\title{
A very Mendelian year
}

We kick off 2022 by noting that the 200th anniversary of Gregor Mendel's birth will be on 20 July 2022. We look forward to celebrating this milestone and reflecting on how far the genetics field has developed in 200 years. Here are some of the things that we are most excited about for 2022.

A s we have previously noted, learning Mendel's laws act as the foundation for an education in genetics, and in the modern era, this must be complemented by studying the latest developments in genomics technologies to have a solid grounding for genetics research. To mark this Mendelian milestone, we review the achievements of the genetics field during these 200 years, and look forward to new things in 2022.

One area of current uncertainty is the ongoing COVID-19 pandemic. It is clear that the past two years have been extremely challenging, on both personal and professional levels, and there does not seem to be a return to a pre-pandemic 'normal' anytime soon. Although we have seen some re-establishment of travel and in-person meetings are increasingly being planned and taking place, the emergence of new variants and uneven vaccination distribution are contributing to the need for continued vigilance and caution. It is incredible to look back at the '19' of COVID-19 from the perspective of 2022 and realize that we are entering the third year of the pandemic.

Although it is unwise to speculate on how the year will shape up in terms of the continued pandemic, we can at least admire the contribution that genetics research has made to understanding the virus and the disease and anticipate further research breakthroughs. Among the most inspiring developments was the rapid establishment of the COVID-19 Host Genetics Initiative, which consists of a broad, international group of collaborators across the genetics field. The sheer size of the undertaking and the speed with which the analysis was done and results published were inspiring. We continue to look forward to more genetic discoveries across diverse ancestry cohorts and the identification of genes that influence susceptibility to COVID-19 or disease severity.

In other areas of genetics research, our current January issue features some content that serves as a great introduction to the new year.

We continue to consider neurogenomics to be an area of high interest, and are excited about the increasing availability of multi-omics resources for brain cells and tissues that are hard to analyze. For example, you can read about a transcriptome and expression quantitative trait loci (eQTL) study of primary human microglia samples isolated at autopsy.

Furthermore, we are keen to see developments surrounding polygenic risk scores (PRS) and methods to generate and evaluate these. In this issue, Ding and colleagues highlight the importance of incorporating uncertainty in estimates of individual PRS into downstream analyses to work towards the goal of clinical applications in precision medicine.

Deep learning is enabling increasingly sophisticated phenotyping of traits that then can be subjected to genome-wide association studies (GWAS). For example, Pirruccello and colleagues trained a deep-learning model based on cardiac magnetic resonance images (data from the UK Biobank) to evaluate the ascending and descending thoracic aorta. We look forward to seeing the continued expansion of health-related phenotypes for GWAS studies and the leveraging of machine learning to explore different tissues and architectures, leading to new biological discoveries.

In addition to human studies, we are eager to observe the plant genomics field and the exciting applications of recent technological genomics developments (for example, long-read sequencing, genome editing, single-cell sequencing and 3D genomics) in this sector. We point you to the sequencing of the lychee fruit genome and a plant genetics study that is appropriate for celebrating this Mendel anniversary year.

Finally, to herald 2022, we would also like to highlight the launch of our new sister journal, Nature Cardiovascular Research. This is a broad scope journal that publishes basic and clinical research on cardiovascular and blood biology, including cardiovascular genetics. To help welcome this new journal, we are happy to feature a News \& Views Article from the Stockholm-Tartu Atherosclerosis Reverse Network Engineering Task (STARNET), which we encourage you to read. We eagerly await the advances that will emerge from all areas of cardiovascular research, particularly genetics, that will be published in Nature Cardiovascular Research.

We wish everyone a positive start to the new year, and hope that you will join us in commemorating the 200th anniversary of Mendel's birth in July. We are looking forward to a great 2022 !

Published online: 12 January 2022 https://doi.org/10.1038/s41588-021-01002-x 